\title{
Melanoma Biomarker
}

National Cancer Institute

\section{Source}

National Cancer Institute. Melanoma Biomarker. NCI Thesaurus. Code C46091.

Skin cancer in melanocytes is termed melanoma, which is frequently centralized in a nevus or mole. A host of molecular proteins can be utilized as measurable phenotypic parameters to characterize or indicate the disease state of melanoma. 2005 